Journal of Computer Science 8 (1): 31-40, 2012

ISSN 1549-3636

(C) 2011 Science Publications

\title{
Information Systems Development Knowledge Sharing-Organizational Justice, Physical Distance and Social Interdependence
}

\author{
Marzanah A. Jabar, Cheah Chee Yeong and Fatimah Sidi \\ Department of Information System, \\ Faculty of Computer Science and Information Technology, \\ University Putra Malaysia, 43400, Serdang, Malaysia
}

\begin{abstract}
Problem statement: Knowledge hoarding in Requirements Elicitation(RE) phase could lead to Information Systems Development (ISD) project failure. Many researchers have discussed important factors in knowledge sharing. Nevertheless, the success rate for ISD projects is low and there are other major factors for consideration. Approach: Review was conducted to identify potential gaps in existing literature and to explore new factors that impact ISD knowledge sharing in RE. Results: Based on systematic review of literature, three new factors are identified namely Organizational Justice, Physical Distance and Social Interdependence. A theoretical model and future research areas are proposed. Conclusion: The success of ISD projects are impacted by organizational justice, physical distance andsocial Interdependence. The proposed model provides project managers a new platform to further understand ISD knowledge sharing between business users and external IT professionals in RE.
\end{abstract}

Key words: Knowledge sharing, information systems development, reasoned action, organizational justice, social interdependence, human capital

\section{INTRODUCTION}

It is predicted that the coming of the knowledge economy in which the knowledge resident in society or organizations would represent value for the organization more than other means of production such as equipment and plant facilities. With the increasing importance of knowledge, the management of human capital and the associated knowledge capital has moved from a secondary role, where it rested until the end of the 20th century, to a primary and central role that, if properly managed, can be a source of competitive advantage and innovation for organizations. As business is no longer confine by national boundaries, it is invaluable to organizations to manage their knowledge and to sharing existing skills, knowledge and expertise effectively within an organization in order to be competitive. In other words, convert individual knowledge to organizational knowledge. From ISD perspective, business users need to share their business requirements and processes with IT professionals hence Information Systems (IS) developed could meet their expectations. On the other hand, IT professional must share the technical features and possibilities of the new IS hence business could realise the potential of the technology. Lacking of knowledge sharing from either side could lead to the new IS fails to meet business objective.

The primary research objective of this study is to explore new factors which impact ISD knowledge sharing in RE. This leads to two research questions:

- What are the existing factors impact ISD knowledge sharing in RE?

- What are the new factors potentially impact ISD knowledge sharing in RE?

Knowledge: Knowledge is a justified belief that increases an entity's capacity for effective action, which requires physical skills and competencies, or cognitive or intellectual activity, or both the competencies and cognitive activity to perform that particular action. In an organizational setting, knowledge comprise all cognitive expectancies-observations that have been meaningfully organised, accumulated and embedded in a context through experience, communication, or influence that an individual or organizational actor uses to interpret situations and to generate activities, behaviour and solutions no matter whether these expectancies are rational or used intentionally.

Corresponding Author: Marzanah A. Jabar, Department of Information System, Faculty of Computer Science and Information Technology, University Putra Malaysia, 43400, Serdang, Malaysia 
Knowledge is often distinguished from information and data. Data is the representation of raw numbers and facts. Once it is systematically processed, organised, or given structure, it become information. When individual view and possess such information into their brain, it is in the process to become knowledge. Knowledge could be broadly grouped into individual knowledge and organizational knowledge. Individual knowledge is knowledge that resides in an individual mind. Organizational knowledge, on the other hand, is knowledge that is formed through interactions between technologies, techniques and people. The pattern and form of interactions depend on an organization's history and culture. Organizational knowledge could be further classified into explicit knowledge and tacit knowledge. Explicit knowledge can be documented and shared through information technologies in the form of data, scientific formula, specifications and manuals. This type of knowledge can migrate in the business community and be accessible for most companies regardless of their cooperative activity. It is codified and stored in databases where it can be accessed and used easily by anyone in the company. Tacit knowledge is the exact opposite of explicit knowledge. Tacit knowledge resides in the human mind, behaviour and perceptions. It is highly idiosyncratic, cognitive, hard to formalise and context-sensitive in nature, making it difficult to communicate and share with others. Examples of tacit knowledge are intuitions, hunches, insights, beliefs and values. From ISD perspective, business requirement is a combination of tacit and explicit knowledge.

Knowledge sharing definitions:Knowledge sharing has received a major attention because it is one of the primary pillars in knowledge management efforts. More organizations are now addressing the issue of knowledge sharing because of their growing awareness of the importance of knowledge to organizational success. Knowledge sharing is a deliberate act in which knowledge is made reusable for one party through its transfer by another. Knowledge sharing is a process taking place between two actors and the process may involve one or more people. It may take place between two people in a one-to-one relationship such as a conversation over a cup of coffee. Or it may be a oneto-many interaction such as in a meeting or a presentation. Knowledge sharing can also be seen as a process of knowledge exchange. It has been argued that the motivation for these different exchanges is related to the expectation of receiving something in return.

In knowledge sharing situations, reciprocal knowledge exchanges instead of one-way knowledge transfers. Nonetheless, even in reciprocal exchanges, each party is at times either a source or a recipient with respect to what they are sending or receiving. Thus knowledge sharing is a process of bidirectional exchange of knowledge. The author define knowledge sharing in this study context as the extent to which business users and IT professionals consciously reveal the presence of knowledge and exchange pertinent knowledge with one another during RE.

The importance and challenges of knowledge sharing: Today and increasingly in the future, in a knowledge age where national boundaries are of less importance to business, the transfer of knowledge and expertise and the creation of a learning organization has become a critical factor to organization success and competitiveness. As companies move forward, they must negotiate difficult paths between serving existing markets and developing new initiatives to meet the challenges of new competitors and opportunities. The key to negotiating between these opposing forces successfully is knowledge, specifically the knowledge assets each firm holds within.

Many countries have an ageing population which implying a greying workforce for many organizations. Drucker (2002) observed that by 2030, people over sixty-five in Germany, the world's third largest economy, will account for half of the adult population, while the population of those under thirty-five will shrink about twice as fast as the older population will grow. These figures are similar to those in several developing countries as well. This implies that more people will be retiring soon with not enough workers of the next generation of workers to replace them and contribute to their retirement incomes through payroll taxes. The retirement of older workers, the failure of companies to retain mid-career employees and the lack of focus on recruiting new employees are main threats to the health and viability of organizations of all shapes and sizes. As the level of experience generally increases with age, in many organizations, older employees are likely to possess more experience and sometimes more knowledge than younger employees. Unfortunately, as these older employees retire, they tend to carry the knowledge they have acquired over many years of practice with them, sometimes creating difficulties for the organizations. Other than through retirement, knowledge can also be lost through redundancy, resignation or promotion.

Sharing of knowledge has to take place in order to take advantage of the knowledge silos that exist in organization, to avoid reinventing the wheel, to reduce duplication and replication of effort and to avoid the 
same errors. However, individuals do not share their knowledge under all circumstances and they have enough reasons not to be willing to share as much as the organization would like them to.

Information Systems Development (ISD): In recent years, organizations have experienced an increasing demand for the development of IS. Research indicates that ISD projects are becoming more ambitious, more organizationally and technically complex and more time to market focused (Reich, 2007). Unfortunately, researchers and practitioners have observed that a large proportion of these ISD projects failed and resulted in significant costs to organizations. According to a survey conducted by research firm Standish Group, United States investment in ISD projects in 2001 was four times that reported in 1990. However, success rate was a mere 28\%. Figures remained troublesome in 2009. ISD project success rate was $32 \%, 44 \%$ of projects were over budget and time estimated and $24 \%$ failed outright Standish Group, 2009. These statistics suggest that many efforts to improve project management barely help to increase success. A high success rate is crucial to technology-backboned modern organizations. Project failures not only waste resources and lead to foregone business opportunities, but impact reputations and profits as well.

Several researchers have mentioned that ISD projects failure were mainly due to inability of the IS to accurately meet user requirements, a consequence of incomplete and inaccurate information requirements collection during the RE phase (Mathiassen et al., 2007). Given the criticality of the RE phase in ISD projects, RE has been and remains, an important domain for IS research. Within this context, knowledge management plays an important role to improve the effectiveness of all primary activities by increased learning ability (Tesch et al., 2009).

\section{MATERIALS AND METHODS}

To align with research question to examine what other attributes impact knowledge sharing in RE, systematic literature review was undertaken to secure insights into the views of various authors about ISD knowledge sharing, particularly factors that affect success and the methodologies used. The process of literature review prevents us from redoing the same study that was already discovered as well as reducing the possibility of repeating the same errors. It also provides an insight on the topic of interest, highlights research gaps and hence, helps us to refine the research questions and objectives respectively. The literature review ensures that no important variables have been ignored.

\section{RESULTS}

Theory of Reasoned Action (TRA): The TRA is intended to predict specific behaviors that individuals have control over, in this case, knowledge sharing practices. Knowledge sharing practices can be studied by applying the theory of reasoned action, wherein attitudes are predicted by evaluating an individual's intention to perform certain behaviors and the available subjective norms (Leibowitz, 2007). Knowledge sharing may lead to an increase in self-esteem that is based on a sense of competence, power and achievement. Hence, people who have positive feelings about sharing their knowledge may be more inclined to do it.

Perceived subjective norms are indicators of people's willingness to comply with others. If an individual employee feels that his colleagues and managers expect him to share his knowledge with them and if he would like to do so, then he also has the intention to share his knowledge. Since people like to be identified and accepted by other members of their organization, perceived subjective norms play a key role in forming their intention to share knowledge (Sun and Scott, 2005).

ISD knowledge sharing: The author empirically examined various literatures on ISD knowledge sharing Table 1. The empirical evidence on the effectiveness of individual and organizational factors in ISD projects knowledge sharing is mixed. There are many other studies from knowledge management perspective that focus on fine-grained transfers of knowledge embedded in technology, routines, people, or subnetworks. The formation of studies on ISD knowledge sharing has investigated numerous factors that impact knowledge sharing success, however, only a few studies have attempted to integrate individual and organizational factors and those that do either are conceptual studies or omit factors identified by others as important.

As individuals are the main stakeholders of knowledge management initiatives, more research should be performed around individual variables and knowledge sharing intention for a better understanding of knowledge sharing in RE phase. Connelly and Kelloway (2003) supported that individual and organizational enablers of knowledge sharing are not properly clarified. Further analysis of how different types of individual and organizational factors and its combined impact affect ISD knowledge sharing in RE phase is absent from the literature. Hence, there are opportunities to bridge this gap by examining these factors in a single study. 
Table 1: Factors related to ISD knowledge sharing in RE

\begin{tabular}{ll}
\hline Area of study & Factors \\
\hline Sender (Knowledge source) & Expertise/capability (Joshi et al., 2007) \\
& Reputation and credibility (Joshi et al., 2007; Ko et al., 2005; Sarker et al., 2005) \\
Receiver (Knowledge recipient) & Encoding competency (Ko et al., 2005) \\
& Absorptive capacity (Ko et al., 2005; Tiwana and McLean, 2005) \\
Motivation & Decoding competency (Ko et al., 2005) \\
& Job Satisfaction (De Vries et al., 2006) \\
Relationship network & Extrinsic and Intrinsic motivation (Ko et al., 2005) \\
& Social Network (Chow and Chan, 2008) \\
Channel & Shared understanding and arduousness of relationship (Ko et al., 2005) \\
Trust & Communities of practice (Yu et al., 2011) \\
& Channel richness (Lind and Zmud, 1991) \\
Communication & Social trust (Chow and Chan, 2008) \\
& Previous experience with trust (Luna-Reyes et al., 2004) \\
Information systems & Shared language (Chang and Chuang, 2011) \\
& Use of systematic language (Bou-Llusar and Segarra-Cipres, 2006) \\
& Information quality (Kuo and Lee, 2009) \\
& Comfort while using knowledge sharing technologies and tools \\
Reward & (Goulding et al., 2007) \\
& Computer-based IS and electronic media (Huysman and Wulf, 2006) \\
Organizational & Effectiveness of knowledge sharing rewards (Chang and Chuang, 2011) \\
& Existence of team-based rewards (Milne, 2007) \\
Cultural & Organization Commitment (De Vries et al., 2006) \\
& Team creativity (Tiwana and McLean, 2005) \\
Personality traits & Ease of information flow (Syed-Ikhsan and Rowland, 2004) \\
Power & National cultures (Minbaeva, 2007) \\
& Collectivism (Hwang and Kim, 2007) \\
& Agreeableness, Conscientiousness, Openness (Matzler et al., 2008) \\
& Big Five analysis (Bove and Mitzifiris, 2007) \\
& Perceived knowledge power (Jayasingam et al., 2010) \\
\hline
\end{tabular}

Looking from communication perspective, knowledge sharing not only depends on the attributes of source, recipient and channel but is also affected by the context within which knowledge sharing takes place. Recognizing this, several researchers have examined the effects of motivation and social relationship on knowledge sharing. In the context of ISD, motivational factors have largely been conceptualized in terms of intrinsic and extrinsic motivations. The nature of social relationships and networks also impact knowledge sharing. Knowledge exchange pairs that have little shared understanding and arduous relationships are less likely to interact and relationships that lack mutual trust hinder knowledge sharing. Future knowledge in the form of data and information can be stored in a variety of ways and could be shared in various ways as well such as via email, groupware, internet, intranet and videoconferencing. In this sense, IT should be seen as a necessary tool, but technology and its use is not of itself knowledge management or indeed knowledge sharing.

In spite of collaboration tools and the advances of technology, "soft" factors such as human relationships, leadership and culture clearly play an important role and may be the main key to further understand the dynamics and effective knowledge sharing in ISD projects. The significance of personal contact should not be underestimated, especially in global ISD projects, where an understanding of cultural differences, such as business behavior, attitudes, mindsets, perceived fairness and language can be critical to ISD projects success. In addition, it is necessary and important to understand that creating and sharing knowledge are intangible activities that can neither be supervised nor forced out of people. It happens only when individuals cooperate voluntarily in an organization with positive environment. Although motivation and expertise might account for individual participation in knowledge sharing (Wang and Lai, 2006), it is not always easy to predict when and why employees share their knowledge. Thus, individual factors are also among those key elements that need to be considered while studying knowledge sharing behavior (Kuo and Young, 2008).

Research in the area of knowledge sharing has been conducted in many settings and for various objectives. However, limited attention has been directed towards examining the role of knowledge sharing in ISD projects (Joshi et al., 2007; Tesch et al., 2009), particularly between business and external IT professionals in RE phase. Engaging external IT professionals or vendors is getting more frequent, with the worldwide IT services market totalling US\$819 
billion in 2008. With the growing trend of organizations outsourced their IT capabilities and services, the problem is even more pronounced for ISD teams involving external IT professionals, where the gap between business and IT professionals is further widened by organizational boundaries (Pee et al., 2010). From the literature review, the author noted that the study on physical distance of ISD team and its impact to effectiveness of RE is lacking. Although some have mentioned co-location of project team but virtual team is more common in today project setup because of the used of collaboration tools. Hence, the importance of physical distance and its impact to RE needs further investigation.

Several researchers have discussed various components of organizational justice namely distributive justice, procedural justice and interactional justice in the domains of job satisfactions, performance management, conflict management, negotiation and virtual communities (Chiu et al., 2011; Das and Kumar, 2010; Farndale et al., 2011; Hassan and Hashim, 2011). These studies demonstrated that organizational justice is an important ingredient to motivate people to contribute to organisation's objective. People need to be treated fairly and with full respect hence, to secure their willingness to contribute. Hence, investigation of organizational justice in ISD is relevant and important as the success of RE is closely related to strong human relationship and their willingness to share knowledge. In addition, different component of organizational justice may have different degree of impact on RE.However, there are limited discussions on the impact of organizational justice specifically in ISD knowledge sharing in RE hence, an area for further investigation.

RE techniques:Regardless of whether the systems development environment utilizes a traditional structured methodology, such as the waterfall model, or a contemporary iterative methodology, such as the 'agile' development model, nearly every identified methodology contains the general activities of analysis, design, code and test, with requirements determination taking place within the analysis activity.

Table 2: Techniques to improve RE effectiveness

\begin{tabular}{ll}
\hline Techniques & Researchers \\
\hline Communication & (Ocker et al., 1998) \\
Interviewing & (Browne and Rogich, 2001; Moody et al., 1998) \\
Problem solving & (Mumford, 2000) \\
Domain & (Marakas and Elam, 1998) \\
Scenario & (Breitman et al., 2005) \\
Modeling & (Marttiin et al., 1995) \\
Design & (Iivari et al., 1998; Lynch and Gregor, 2004) \\
Viewpoint & (Sommerville, 2006) \\
GSS-aided JAD sessions & (Liou and Chen, 1993) \\
Cognitive mapping & (Siau and Tan, 2006) \\
\hline
\end{tabular}

Three stages of requirements determination are: (1) information gathering, during which the analyst elicits requirements from stakeholders about needs for the proposed system; (2) representation, during which the requirements are represented in a physical form and (3) verification, during which the requirements are verified with stakeholders. This study is concerned with information gathering, or what is often referred to as requirements elicitation. Enormous research has been done to address the challenges in RE. These literatures covered various techniques to improve RE effectiveness Table 2. In spite of these fruitful studies, many limitations have been identified by scholars (Chakraborty et al., 2010).

The ability to elicit requirements effectively depends in part on familiarity with a domain. Strong problem-solving skills are also critical for successful requirements elicitation. These skills and domain knowledge assist the project manager in structuring the problem, acquiring and organizing information and transforming that information into a complete and accurate set of system requirements. However, even sufficient domain knowledge and strong problemsolving skills do not guarantee RE success. Business users and IT professionals are subject to cognitive limitations that can hamper or prevent adequate RE (Pitts and Browne, 2007).

One of the most acclaimed methods for system specification is JAD (Joint Application Development). JAD is a generic term that describes a variety of methods for conducting workshops in which stakeholders and developers work together on the system development phases, including requirements definition. The main approach of JAD is the use of group dynamic techniques to facilitate the workshop sessions. The technique has been widely used but so far there are no concluding studies about the efficacy of the method in generating accurate and complete system requirements. Scenarios have been shown to be very helpful in identifying and communicating requirements. New software development techniques, such as XP (Extreme Programming), use scenario descriptions and try to identify how easily users communicate using these scenarios. However, they do not fully use the collaboration concept. Although XP groups collect different viewpoints, the analyst is the one responsible for deciding the criteria to be used in this grouping. This may lead to erroneous interpretation of requirements. Viewpoint oriented techniques consider that each stakeholder may see the future system under a different perspective and to capture different viewpoints of the system. An example of such technique is the VORD (Viewpoint Oriented Requirements Definition) technique. It identifies and structures the viewpoints, but it does this in a non-collaborative way as only the 
analyst carries out the requirements integration. In essence, the main gap of these techniques is under estimating the importance of social interdependency between business users and analysts during RE. A silo view and assumptions of the analysts caused wrong interpretation and integration of business requirements.

ISD is a knowledge-intensive process encompassing requirements as to gathering, design, implementation, testing, deployment, maintenance and project coordination and management activities. Essentially, with business and IT knowledge being the most critical components (Pee et al., 2010). Many studies have highlighted the important role of rewards and goals but not many have discussed specifically from the angle of perceived goal and reward interdependence and its impact to effective ISD knowledge sharing in RE.Viewing RE from the theory of social interdependence, knowledge regarding business requirements, processes and workflow are needed to provide the logic driving the new IS. On the other hand, IT knowledge related to the use and technical possibilities of the new system is needed by business users to fully realize the potential of the new IS. This understanding would prevent analysts study in silo and dictate business requirements wrongly but encourage them to work handin-hand with business users to ensure the new IS meeting all requirements and objectives of both parties.

To summarize from the systematic literature review, in spite of various literatures and techniques to improve ISD knowledge sharing in RE, three new factors are found and might have significant impact to the dimension of this study. These new factors are organizational justice, physical distance and social interdependency which have been lightly mentioned in existing literatures. They deserve further investigations and would provide greater insights on their impact to effectiveness of ISD knowledge sharing in RE between business users and external IT professionals.

\section{DISCUSSION}

Theoretical model:Knowledge sharing is not an arbitrary activity that occurs independent of other activities within an organization, but one that are affected by questions of what is being transferred and how the transfer will occur.

With the understanding and arguments presented in prior research, the author proposed a theoretical model based on the three new factors identified in systematic literature review and grouped it under individual and organizational categories respectively Fig. 1.

Organizational factors:Organizational justice is considered to encompass three different components; distributive justice, procedural justice and interactional justice.

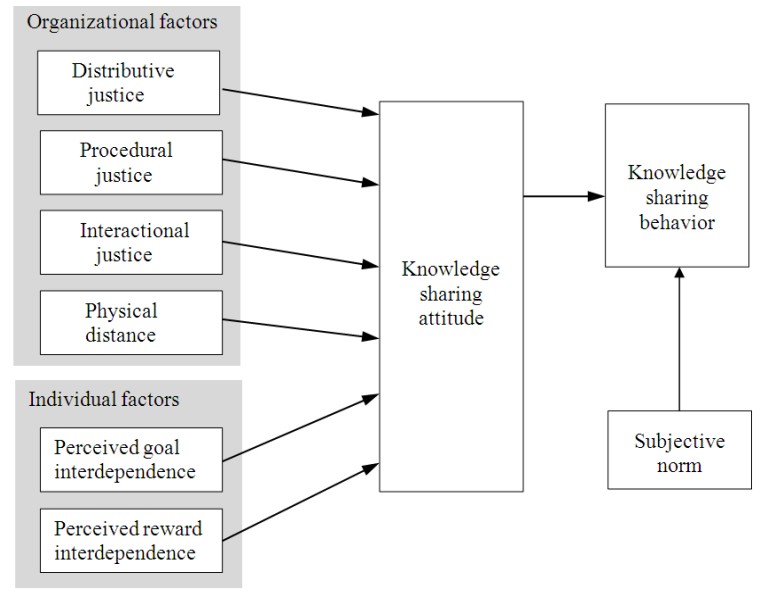

Fig. 1: Theoretical Model of ISD knowledge sharing in RE phase

Distributive justice refers to the fairness of outcomes received. Individuals will evaluate distributions of outcomes with respect to some distributive rule, the most common of that is equity. Equity assessment involves a comparison of one's inputs and obtained outcomes relative to a referent comparison other. Inequity is hypothesized to exist when both more and less outcomes are received than were expected. The equity distribution rule suggests that people should receive rewards that are consistent with the inputs they contribute to a distribution situation. Evaluations of inequitable distributions are thought to produce negative emotions and increase perceptions of unfairness. Bock et al. (2005) found that anticipated extrinsic rewards hindered creation of positive attitudes toward knowledge sharing. They attributed this surprise finding to differences between management and employees in their perceptions of appropriate extrinsic rewards. Another explanation might be that management and employees disagreed on the fairness of the distribution of rewards thus negatively affecting attitudes. In other words, a fair distributive justice perception may encourage ISD knowledge sharing in RE.

Procedural justice refers to an exchange between an organization and its employees. Procedural justice has to do with following procedures that are consistent, unbiased, accurate, correctable, representative and ethical. Although employees' reactions toward an organization have been studied extensively relatively little is known about how procedural justice might affect business users and IT professionals' intention to share their knowledge during RE phase. If the organizational policies and procedures are perceived as 
fair and equitable, organizational citizenship behavior will be enhanced and they may be more likely to have positive attitudes towards sharing their expertise. Regardless of how the rewards are distributed, perceptions of bias in the evaluation process will lead to decrease in the procedural justice perceptions and produce negative knowledge sharing attitudes.

Exchange theorists suggest that human interactions are characterized by social economics, where people are concerned about the inputs they invest in relationships and the outcomes they receive from these relationships. Reciprocal relationship is an important determinant of attitude towards knowledge sharing. We argue that this construct can be viewed through the lens of the interactional justice. Items such as "My knowledge sharing would draw smooth cooperation from outstanding members in the future" from Bock et al. (2005) also indicate that employees expect reciprocity when sharing their knowledge, further reflecting employee beliefs that knowledge sharing will lead to the improvement of their relationships with others. Both of these inferences are supported by interactional justice. If individuals treat each other with dignity and respect, a sense of mutual trust and openness will preclude knowledge hoarding behavior.

Physical distance refers to the difficulty, time requirement and expense of communication and getting together face-to-face. Davenport and Prusak (1998) highlighted that sometimes knowledge sharing can only work if the various parties are brought together physically, in other words, face-to-face communication. To lower disadvantages of bureaucracy and formal communication, modern shop and office layout reduce the distance between worker and executives to foster ad hoc, informal and face-to-face communication. Intel accelerated the development process for microprocessors and ensured the quick harvesting of research results by collocating the process development and production groups to facilitate exchange of ideas and to enable the groups to gain an understanding of the issues they faced. From ISD perspective, co-locate the design team may improve RE effectiveness.

Individual factors: In addition to project goals, business users and external IT professionals generally have their own goals in mind. This is mainly due to these subgroups have different backgrounds, expectations, skill sets and roles in the ISD project. When the subgroups' goals are perceived as interdependent, they will tend to promote their mutual goal attainment by coordinating and cooperating with each other. For instance, the business users may seek to develop a system that can meet business needs and target to complete the project on time and within budget. On the other hand, the external IT professionals may endeavour to develop a good quality IS and employ the latest technology to diversify their portfolio. In this case, the first goals of the two subgroups are largely interdependent, as the business counts on the expertise of external IT professionals to build the IS and the external IT professionals relies on the judgment of business to evaluate the quality of the developed IS. Awareness of this interdependence can induce the subgroups to work jointly to achieve their mutually supporting goals.

Studies of conventional teams have shown that carefully implemented rewards can foster team spirit, enhance members' willingness to contribute to the team's success and facilitate knowledge sharing (Siemsen et al., 2007). If the subgroups are aware that their rewards are contingent upon the other subgroup's performance, they may be more willing to share knowledge with the other subgroup when requested to maximize their collective rewards.

Future research:The proposed model is in its conceptual stage. Future works would be on developing relevant hypotheses, data collection, analysis and to validate the proposed hypotheses. Proving using formal method may be useful. This study has identified several factors found to contribute to ISD knowledge sharing. However, the factors used in this study are in no way exhaustive and hence, the theoretical model has been underspecified. Other interesting factors related to knowledge sharing are importance of corporate image, commitment, language competency, psychological effect and self interest. Although these factors have not been addressed explicitly in this study, they may need to be considered in future research. Future research may also consider applying the model to study other important phenomenon such as global virtual collaboration and offshore outsourcing. Moreover, the propositions that are presented need to be empirically tested. This study identified many factors which impact effectiveness of RE. Exploratory study to rank the importance of these factors to ISD knowledge sharing in RE could be useful as it facilitate project managers to focus their effort on main factors. As organizations are looking forward to cut cost and to outsource non-core businesses, there will be an increased emphasis on employment of contingent employees such as in the area of IT. The use of contingent employees on shortterm and long-term assignments will have different consequences for the pursuit of any ISD knowledge sharing objectives by the organizations. Consequently, this situation needs to be examined further, especially in knowledge-oriented organizations. For instance, how the tenure of contingent employees affects the achievement 
of knowledge sharing goals needs to be investigated. Overall, this study will serve as a point of departure for future research on ISD knowledge sharing in RE.

\section{CONCLUSION}

Systematic review was conducted on literature related to ISD knowledge sharing in RE. The identified attributes, models and techniques are tabulated for detailed discussions. The research objective was met with three new factors found (organizational justice, physical distance and social interdependency). Based on these new factors, a theoretical model is proposed to provide project managers a new platform to further understand ISD knowledge sharing between business users and external IT professionals in RE. Future research areas are highlighted for consideration.

\section{REFERENCES}

Bock, G.W., R.W. Zmud, Y. Kim and J.N. Lee, 2005. Behavioral intention formation in knowledge sharing: examining the roles of extrinsic motivators, social-psychological forces and organizational climate. MIS Q., 29: 87-111.

Bou-Llusar, J.C. and M. Segarra-Cipres, 2006. Strategic knowledge transfer and its implications for competitive advantage: An integrative conceptual framework. J. Knowledge Manage., 10: 100-112. Retrieved from DOI: 10.1108/13673270610679390

Bove, L. and B. Mitzifiris, 2007. Personality traits and the process of store loyalty in a transactional prone context. J. Services Mark., 21: 507-519. DOI: 10.1108/08876040710824861

Breitman, K.K., J.C.S. Do Prado Leite and D.M. Berry, 2005. Supporting scenario evolution. Requirements Eng., 10: 112-131. DOI: 10.1007/s00766-004-0199$\mathrm{z}$

Browne, G.J. and M.B. Rogich, 2001. An empirical investigation of user requirements elicitation: comparing the effectiveness of prompting techniques. J. Manage. Information Syst., 17: 223249.

Chakraborty, S., S. Sarker and S. Sarker, 2010. An exploration into the process of requirements elicitation: A grounded approach. J. Association Infor. Syst., 11: 212-249.

Chang, H.H. and S.S. Chuang, 2011. Social capital and individual motivations on knowledge sharing: Participant involvement as a moderator. Inf. Manag., 48: 9-18. DOI: 10.1016/j.im.2010.11.001
Chiu, C.M., E.T.G. Wang, F.J. Shih and Y.W. Fan, 2011. Understanding knowledge sharing in virtual communities: An integration of expectancy disconfirmation and justice theories. Online Inf. Rev., $\quad 35$ : $134-153 . \quad$ DOI: $10.1108 / 14684521111113623$

Chow, W.S. and L.S. Chan, 2008. Social network, social trust and shared goals in organizational knowledge sharing. Inf. Manage., 45: 458-465. DOI: $10.1016 / j . i m .2008 .06 .007$

Connelly, C.E. and K. Kelloway, 2003. Predictors of employees' perceptions of knowledge sharing cultures. Leadership Organization Development J., 24: 294-301. DOI: 10.1108/01437730310485815

Das, T.K. and R. Kumar, 2010. Interpretive schemes in cross-national alliances: Managing conflicts and discrepancies. Cross Cultural Manage.: Inter. J., 17: 154-169. DOI: 10.1108/13527601011038723

Davenport, T.H. and L. Prusak, 1998. Working Knowledge: How Organizations Manage What They Know. 1st Edn., Harvard Business Press, Boston, ISBN: 0875846556, pp: 199.

De Vries, R.E., B. van den Hooff and J.A. de Ridder, 2006. Explaining Knowledge Sharing: The role of team communication styles, job satisfaction and performance beliefs. Commun. Rese., 33: 115-135. DOI: $10.1177 / 0093650205285366$

Drucker, P.F., 2002. Managing in the next society. 1st Edn., Elsevier, New York, ISBN: 0312289774, pp: 321.

Farndale, E., V. Hope-Hailey and C. Kelliher, 2011. High commitment performance management: the roles of justice and trust. Personnel Rev., 40: 5-23. DOI: $10.1108 / 00483481111095492$

Goulding, J., M. Sexton, X. Zhang, M. Kagioglou and G.F. Aouad et al., 2007. Technology adoption: breaking down barriers using a virtual reality design support tool for hybrid concrete. Construct. Manage. Econ., 25: 1239-1250.

Hassan, A. and J. Hashim, 2011. Role of organizational justice in determining work outcomes of national and expatriate academic staff in Malaysia. Inter. J. Commerce Manage., 21: 82-93. DOI: 10.1108/10569211111111711

Huysman, M. and V. Wulf, 2006. IT to support knowledge sharing in communities, towards a social capital analysis. J. Infor. Technol., 21: 4051. DOI: $10.1057 /$ palgrave.jit.2000053

Hwang, Y. and D.J. Kim, 2007. Understanding affective commitment, collectivist culture and social influence in relation to knowledge sharing in technology mediated learning. IEEE Trans. Profession Commun., 50: 232-248. DOI: 10.1109/TPC.2007.902664 
Iivari, J., R. Hirschheim and H.K. Klein, 1998. A paradigmatic analysis contrasting information systems development approaches and methodologies. Infor. Syst. Rese., 9: 164-193.

Jayasingam, S., M.A. Ansari and M. Jantan, 2010. Influencing knowledge workers: The power of top management. Industrial Manage. Data Syst., 110: 134-151. DOI: 10.1108/02635571011008443

Joshi, K.D., S. Sarker and S. Sarker, 2007. Knowledge transfer within information systems development teams: Examining the role of knowledge source attributes. Decision Support Syst., 43: 322-335. DOI: $10.1016 /$ j.dss.2006.10.003

Ko, D., L.J. Kirsch and W.R. King, 2005. Antecedents of knowledge transfer from consultants to clients in enterprise system implementations. MIS Q., 29: 59-85.

Kuo, F.Y. and M.L. Young, 2008. Predicting knowledge sharing practices through intention: A test of competing models. Computers Human Behavior, 24: 2697-2722. DOI: 10.1016/j.chb.2008.03.015

Kuo, R.Z. and G.G. Lee, 2009. KMS adoption: The effects of information quality. Manage. Decision, 47: 1633-1651. DOI: 10.1108/00251740911004727

Leibowitz, J., 2007. Social networking: The essence of innovation. 1st Edn., Scarecrow Press, Lanham, ISBN: 0810858576, pp: 130.

Lind, M.R. and R.W. Zmud, 1991. The influence of a convergence in understanding between technology providers and users on information technology innovativeness. Org. Sci., 2: 195-217.

Liou, Y.I. and M. Chen, 1993. Using group support systems and joint application development for requirements specification. J. Manage. Inf. Syst., 10: $25-41$.

Luna-Reyes, L.F., A.M. Cresswell and G.P. Richardson, 2004. Knowledge and the development of interpersonal trust: A dynamic model. Proceedings of the 37th Hawaii International Conference on System Sciences, Jan. 5-8, IEEE Xplore Press, Mexico, 1-12. DOI:10.1109/HICSS.2004.1265241

Lynch, T. and S. Gregor, 2004. User participation in decision support systems development: Influencing system outcomes. Eur. J. Inf. Syst., 13: 286-301. DOI: $10.1057 /$ palgrave.ejis.3000512

Marakas, G.M. and J.J. Elam, 1998. Semantic structuring in analyst acquisition and representation of facts in requirements analysis. Inf. Syst. Res., 9: 37-63. DOI: 10.1287/isre.9.1.37

Marttiin, P., K. Lyytinen, M. Rossi and V. Tahvanainen, 1995. Modeling requirements for future CASE: Modeling issues and architectural considerations. Inf. Resources Manage., J., 8: 1-15.
Mathiassen, L., T. Saarinen, T. Tuunanen and M. Rossi, 2007. A contigency model for requirements development. Georgia State University.

Matzler, K., B. Renzl, J. Müller, S. Herting and T. A. Mooradian, 2008. Personality traits and knowledge sharing. J. Economic Psychol., 29: 301-313. DOI: 10.1016/j.joep.2007.06.004

Milne, P., 2007. Motivation, incentives and organisational culture. J. Knowledge Manage.,11: 28-38. DOI: 10.1108/13673270710832145

Minbaeva, D.B., 2007. Knowledge transfer in multinational corporations. Manage. Int. Rev., 47: 567-593. DOI: $10.1007 / \mathrm{s} 11575-007-0030-4$

Moody, J.W., J.E. Blanton and P.H. Cheney, 1998. A theoretically grounded approach to assist memory recall during information requirements determination. J. Manage. Inf. Syst., 15: 79-98.

Mumford, E., 2000. A socio-technical approach to systems design. Requirements Eng., 5: 125-133. DOI: $10.1007 /$ PL00010345

Ocker, R., J. Fjermestad, S.R. Hiltz and K. Johnson, 1998. Effects of four modes of group communication on the outcomes of software requirements determination. J. Manage. Inf. Syst., 15: 99-118.

Pee, L.G., A. Kankanhalli and H.W. Kim, 2010. Knowledge sharing in information systems development: A social interdependence perspective. J. Association Information Syste.,11: $550-575$.

Pitts, M.G. and G.J. Browne, 2007. Improving requirements elicitation: an empirical investigation of procedural prompts. Inf. Syst. J., 17: 89-110. DOI: $10.1111 / \mathrm{j} .1365-2575.2006 .00240 . x$

Reich, B.H., 2007. Managing knowledge and learning in IT projects: A conceptual framework and guidelines for practice. Project Manage. J., 38: 517.

Sarker, S., D.B. Nicholson and K.D. Joshi, 2005. Knowledge transfer in virtual systems development teams: an exploratory study of four key enablers. IEEE Transactions Professional Commun., 48: 201-218. DOI: 10.1109/TPC.2005.849650

Siau, K. and X. Tan, 2006. Using cognitive mapping techniques to supplement UML and UP in information requirements determination. J. Comp. Inf. Syst., 47: 59-66.

Siemsen, E., S. Balasubramanian and A.V. Roth, 2007. Incentives that induce task-related effort, helping and knowledge sharing in workgroups. Manage. Scie., 53: 1533-1550. DOI: $10.1287 / \mathrm{mnsc} .1070 .0714$ 
Sommerville, I., 2006. Software Engineering. 8th Edn., Addison-Wesley, Harlow, ISBN: 0321313798, pp: 840.

Sun, P.Y.T. and J.L. Scott, 2005. An investigation of barriers to knowledge transfer. J. Knowledge Manage., $\quad 9$ : 75-90. DOI: 10.1108/13673270510590236

Syed-Ikhsan, S.O.S. and F. Rowland, 2004. Knowledge management in a public organization: A study on the relationship between organizational elements and the performance of knowledge transfer. J. Knowledge Manage., 8: 95-111. Retrieved from DOI: $10.1108 / 13673270410529145$

Tesch, D., M.G. Sobol, G. Klein and J.J. Jiang, 2009. User and developer common knowledge: Effect on the success of information system development projects. Int. J. Project Manage., 27: 657-664. DOI: DOI: 10.1016/J.IJPROMAN.2009.01.002
Tiwana, A. and E.R. McLean, 2005. Expertise integration and creativity in information systems development. J. Manage. Inf. Syst., 22: 13-43.

Wang, C.C. and C.Y. Lai, 2006. Knowledge contribution in the online virtual community: Capability and motivation. Knoledge Sci. Eng. Manage., 4092: 442-453. DOI: 10.1007/11811220_37

Yu, J., Z. Jiang and H.C. Chan, 2011. The influence of sociotechnological mechanisms on individual motivation toward knowledge contribution in problem-solving virtual communities. IEEE Trans. Professional Commun., 54: 152-167. DOI: 10.1109/TPC.2011.2121830 